\title{
Extraction of coupling matrix of Generalized Chebyshev filter Based on MATLAB
}

\author{
Yalin Guan ${ }^{1, a}$, Pei Li ${ }^{2, b}$ \\ ${ }^{1}$ Department of Information Engineering, Communication University of China, Beijing \\ ${ }^{2}$ Department of Information Engineering, Communication University of China, Beijing \\ a ylguan@cuc.edu.cn, ${ }^{b}$ Ipei001@qq.com
}

Keywords: coupling matrix, generalized Chebyshev filter, cross-coupling transmission zero Abstract. In this paper we present a piece of software of extracting coupling matrix of generalized chebyshev filter based on MATLAB. Our software can obtain coupling matrix of generalized chebyshev filter through index which has been given. In order to obtain the coupling matrix, a method is presented that the transfer and reflection coefficients are expressed by N-polynomials. Then the frequency response of this filter is derived and further more the the coupling matrix is extracted. We present a method which can validate the correctness of our software directly using Ansoft Designer.

\section{Introduction}

With the rapid development of the communication technology, index requirements for filters and multiplexers of the base station are more and more high, which means filters require characteristics of lower insertion loss, higher frequency selectivity, linear phase, and so on. In Structure andDesign of Modern Filters, various types of filter structure are very detailed introduced, but with the increasing congestion of the frequency spectrum, the development of filters also need a higher demand. Generalized chebyshev function is very useful and strictly, it can be used to design filters which index is more strictly. It also can be used to design filters with cross coupling. Designing microwave filters with method of synthesis, it is very important to determine structure of network and coupling matrix. A.E.Atia and A.E.Williams proposed a classical method of extracting the cross coupling matrix, and published it in 1974 on MTT [1-2]. H.C.Bell proposed another method which called RCJ's method in 1982 [3], and R.J.Cameron made improvements to RCJ's method in 1999 [4]. In 2003, R.J.Cameron proposed a orthogonal matrix method which is more simple [5]. Our software can extracting $\mathrm{N}$ order filter's coupling matrix by using orthogonal matrix method, and it can add 8 finite transmission zeros at most.

\section{Principle of the Software}

\section{Generalized Chebyshev function}

For an arbitrary two port lossless network which consisting of $\mathrm{N}$ series resonators, its transmission coefficient and reflection coefficient can be expressed as two $\mathrm{N}$ order polynomials:

$$
\mathrm{S}_{11}(\Omega)=\frac{F(\Omega)}{\mathrm{E}(\Omega)} \quad, \quad \mathrm{S}_{21}(\Omega)=\frac{\mathrm{P}(\Omega)}{\varepsilon \mathrm{E}(\Omega)}
$$

Where

$$
\varepsilon=\left.\frac{1}{\sqrt{10^{0.1 \mathrm{~A}_{\mathrm{p}}}-1}} \frac{\mathrm{P}(\Omega)}{\mathrm{F}(\Omega)}\right|_{\Omega=1}
$$

" $\Omega$ " in the formula is the normalization frequency.

The transfer function of filters can be expressed as follow:

$$
\mathrm{H}_{\mathrm{F}}(\Omega)=\frac{1}{\varepsilon \mathrm{S}_{21}(\Omega)}
$$


For a $\mathrm{N}$ order filter, each of $\mathrm{E}(\mathrm{s})$ and $\mathrm{F}(\mathrm{s})$ is a $\mathrm{N}$ order polynomial. For a filter with $\mathrm{n}_{\mathrm{fz}}$ finite transmission zeros, $\mathrm{P}(\mathrm{s})$ is a $\mathrm{n}_{\mathrm{fz}}$ order polynomial.

Define

$$
\mathrm{C}_{\mathrm{N}}(\Omega)=\frac{\mathrm{F}(\Omega)}{\mathrm{P}(\Omega)}
$$

Because it has $\mathrm{S}_{11}^{2}+\mathrm{S}_{21}^{2}=1$, so we can obtain

$$
\mathrm{S}_{21}^{2}(\Omega)=\frac{1}{1+\varepsilon^{2} \mathrm{C}_{\mathrm{N}}^{2}(\Omega)}
$$

Generalized chebyshev function is defined as follow:

$$
\mathrm{C}_{\mathrm{N}}(\Omega)=\cosh \left[\sum_{\mathrm{n}=1}^{\mathrm{N}} \cosh ^{-1}\left(\mathrm{x}_{\mathrm{n}}\right)\right]
$$

Where

$$
\mathrm{x}_{n}=\frac{\Omega-1 / \Omega_{n}}{1-\Omega / \Omega_{n}}
$$

If all transmission zeros are infinite, then $C_{N}(\Omega)=\cosh \left(n \cosh ^{-1} \Omega\right)$ is traditional chebyshev function.

Generalized Chebyshev function has a few advantages. Firstly, it can has arbitrary $\mathrm{N}$ transmission zeros; secondly, there is characteristics of equal ripples in band, and left and right of out band it can be asymmetric due to the arbitrary of transmission zeros.

Finally, we can obtain the function as follow:

$$
C_{N}(\Omega)=\frac{1}{2}\left[\frac{\prod_{n=1}^{N}\left(c_{n}+d_{n}\right)+\prod_{n=1}^{N}\left(c_{n}-d_{n}\right)}{\prod_{n=1}^{N}\left(1-\Omega / \Omega_{n}\right)}\right]
$$

Where

$$
\mathrm{c}_{\mathrm{n}}=\Omega-1 / \Omega_{\mathrm{n}} \quad \mathrm{d}_{\mathrm{n}}=\Omega^{\prime} \sqrt{\left(1-1 / \Omega_{\mathrm{h}}^{2}\right)} \quad \Omega^{\prime}=\sqrt{\left(\Omega^{2}-1\right)}
$$

Then recursion formula of generalized chebyshev function can be obtained:

$$
\operatorname{Num}\left[\mathrm{C}_{\mathrm{N}}(\Omega)\right]=\mathrm{F}(\Omega)=\frac{1}{2}\left[\mathrm{G}_{\mathrm{N}}(\Omega)+\mathrm{G}_{\mathrm{N}}^{\prime}(\Omega)\right]
$$

Where

$$
\mathrm{G}_{\mathrm{N}}(\Omega)=\prod_{\mathrm{n}=1}^{\mathrm{N}}\left(\mathrm{c}_{\mathrm{n}}+\mathrm{d}_{\mathrm{n}}\right) \quad, \quad \mathrm{G}_{\mathrm{N}}^{\prime}(\Omega)=\prod_{\mathrm{n}=1}^{\mathrm{N}}\left(\mathrm{c}_{\mathrm{n}}-\mathrm{d}_{\mathrm{n}}\right)
$$

\section{Coupling matrix}

$\mathrm{K}$ and $\mathrm{J}$ converter can convert a bandpass filter network with both series and parallel resonance circuit to a bandpass filter network with series or parallel resonant circuit. Fig. 1 is a bandpass filter network with J converter. And it has cross coupling. Using Kirchhoff's law, the circuit equation of the bandpass filter network is as follow: 


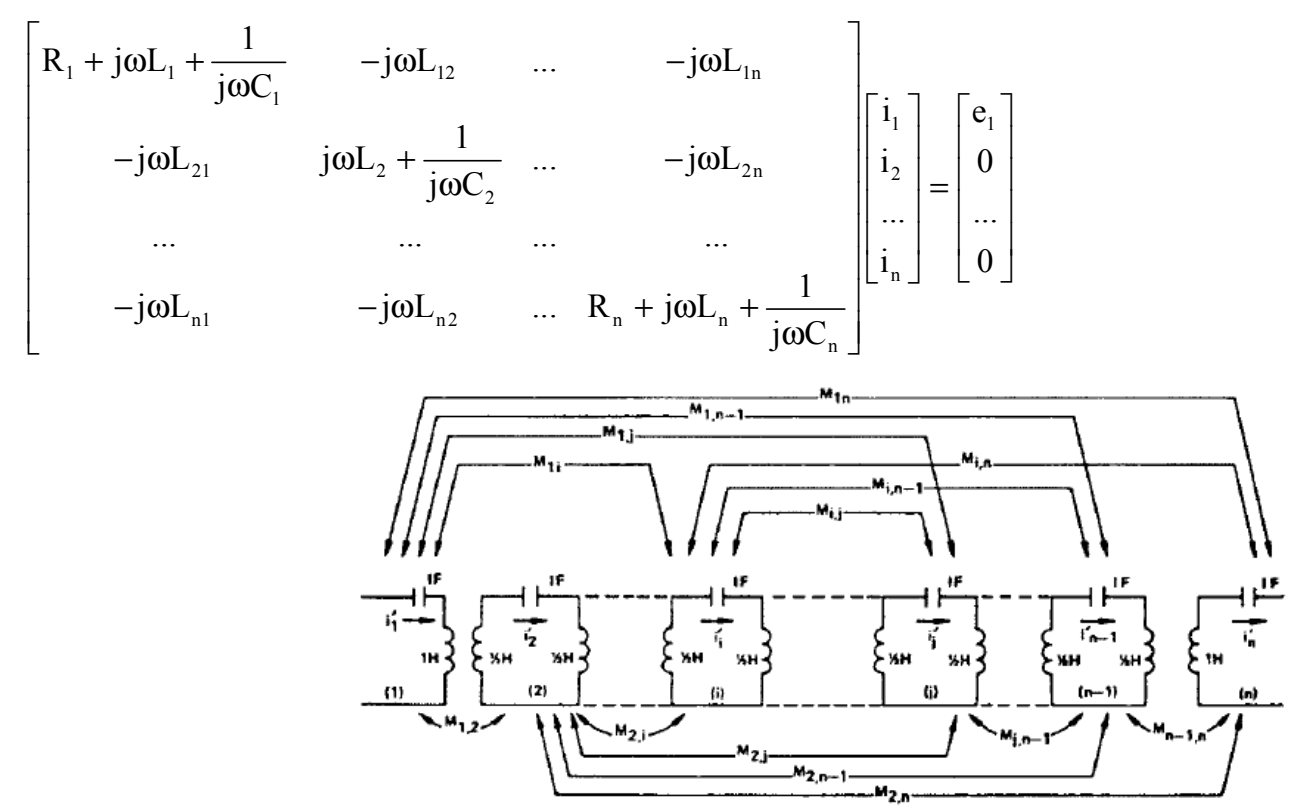

Fig. 1 Bandpass filter network with J converter

It can be written simply as $[\mathrm{Z}][\mathrm{i}]=[\mathrm{e}]$.

[Z] can be written as:

$[\mathrm{Z}]=\omega_{0} \mathrm{FBW} \cdot \operatorname{diag}\left[\sqrt{\mathrm{L}_{1}}, \sqrt{\mathrm{L}_{2}}, \cdots \sqrt{\mathrm{L}_{\mathrm{n}}}\right] \cdot[\overline{\mathrm{Z}}] \cdot \operatorname{diag}\left[\sqrt{\mathrm{L}_{1}}, \sqrt{\mathrm{L}_{2}}, \cdots \sqrt{\mathrm{L}_{\mathrm{n}}}\right]$.

Where

$$
\begin{aligned}
& \text { FBW }=\Delta \omega / \omega_{0},[\bar{Z}]=j p[I]+[q]-j[M], \\
& \mathrm{p}=\frac{1}{\text { FBW }}\left[\omega\left(\frac{1}{\omega_{0}}-\frac{1}{\omega_{\mathrm{k}}}\right)-\frac{1}{\omega}\left(\omega_{0}-\omega_{\mathrm{k}}\right)\right],
\end{aligned}
$$

[I] is unit matrix, $[\mathrm{q}]$ is normalized $\mathrm{Q}$ value, and $[\mathrm{M}]$ is normalized coupling matrix.

$$
[\mathrm{M}]=\left[\begin{array}{cccc}
\mathrm{m}_{11} & \mathrm{~m}_{12} & \ldots & \mathrm{m}_{1 \mathrm{~N}} \\
\mathrm{~m}_{12} & \mathrm{~m}_{22} & \ldots & \mathrm{m}_{2 \mathrm{~N}} \\
\ldots & \ldots & \ldots & \ldots \\
\mathrm{m}_{1 \mathrm{~N}} & \mathrm{~m}_{2 \mathrm{~N}} & \ldots & \mathrm{m}_{\mathrm{NN}}
\end{array}\right],
$$

Where

$$
\mathrm{m}_{\mathrm{ik}}=\frac{\mathrm{M}_{\mathrm{ik}}}{\mathrm{FBW}}, \mathrm{M}_{\mathrm{ik}}=\frac{\mathrm{L}_{\mathrm{ik}}}{\sqrt{\mathrm{L}_{\mathrm{i}} \mathrm{L}_{\mathrm{k}}}}, \mathrm{m}_{\mathrm{kk}}=-\frac{1}{\mathrm{FBW}}\left[\omega\left(\frac{1}{\omega_{0}}-\frac{1}{\omega_{\mathrm{k}}}\right)-\frac{1}{\omega}\left(\omega_{0}-\omega_{\mathrm{k}}\right)\right] \text {. }
$$

After getting $[\mathrm{M}]$, we need to carry on the similar transformation to $[\mathrm{M}]$, and different matrix structure corresponding to different network topology. In this paper, we need to transform it into a folded topology.

\section{Introduction of Software interface}

Software interface is shown in Fig.2. It can be divided into three parts. The first part is input area. In this part, users can input relative index parameters of the filter. Users can input order of the filter, and it can be input 10 order at most. Users also can input frequency center, frequency span, return loss. unloaded $\mathrm{Q}$ value and eight finite transmission zero at most. The second part can display scattering parameters' curve. You can adjust the coordinates you want to see. In the third part, users can obtain $[\mathrm{M}]$, and they can observe the changes of scattering parameters' curve by adjusting the value of [M]. "Adjust Step" expresses the step size of adjusting lengths. 




Fig. 2 Interface of the software

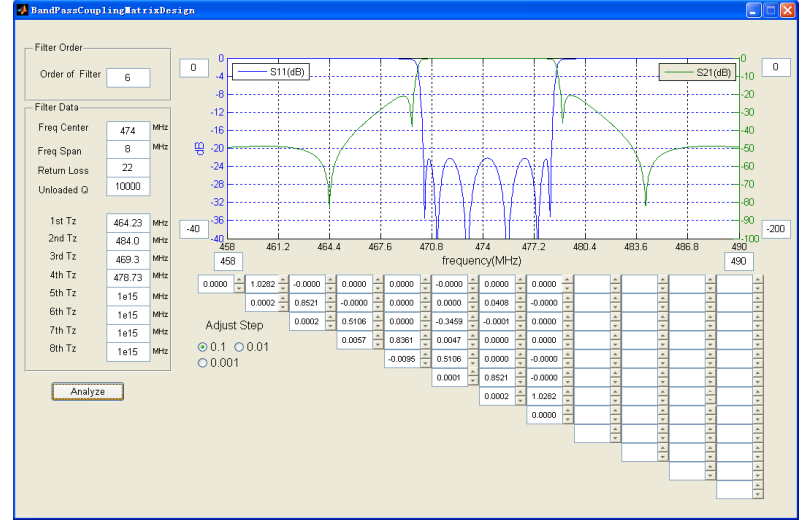

Fig. 3 Interface of the software

\section{Correctness verification}

After introducing the principle and interface of the software, we need to validate the correctness of our software now.

Fig.3 (a) is the result of six orders' filter by using our software. We added two finite transmission zero and the index and result is shown as Fig.3. Then we built up the circuit model by using the software of Ansoft Designer, and it shown as Fig.4 (a). This circuit model can directly using [M]. Fig.4 (b) is the simulation result of Ansoft Designer.

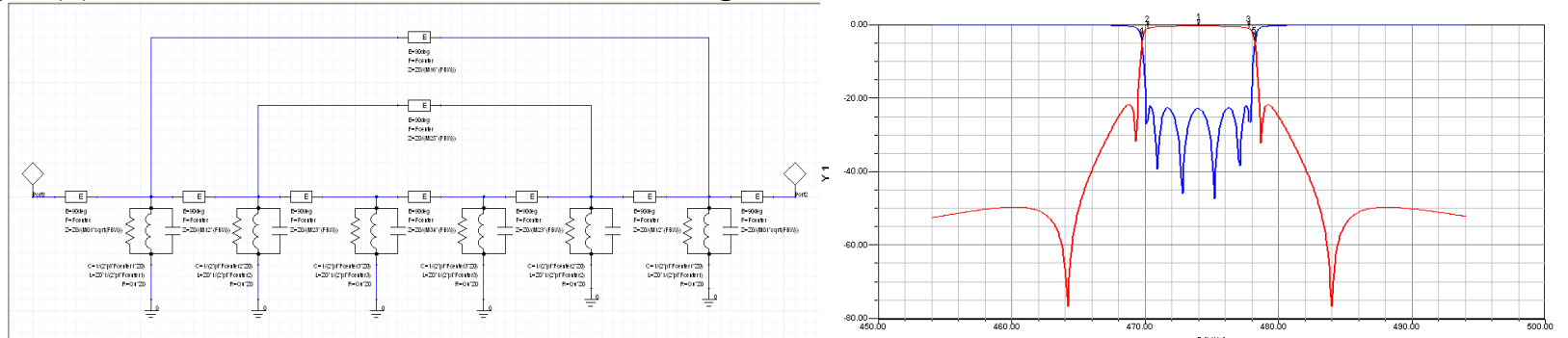

Fig. 4(a) Circuit model by using Ansoft Designer Fig. 4(b) Simulation result of Ansoft Designer

We can find the simulation results of our software are basically similar with that by using Ansoft Designer. That means our software is correct.

\section{Summary}

In this paper, we design a extracting coupling matrix analysis software based on MATLAB. It can simulate ten order's [M] at most. We use Ansoft Designer to validate the correctness of our software. The software has a very high practical value.

\section{References}

[1] Atia A E, Williams A E, New types of waveguide bandpass filters of satellite transponder, J. Comsat Tech Rev.1971, 1(1):21-43.

[2] Atia A E, Williams A E, R W Newcomb, Narrow-band multiple-coupled cavity synthesis, J. IEEE Tans Circuits Syst,1974,21(5):649-655.

[3] H C bell, JR. Canonical asymmetric coupled-resonator filters, J. IEEE Tans Microwave Theory Tech,1982,30(9):1335-1340.

[4] Cameron R J. General coupling matrix synthesis methods for Chebyshev Filtering functions, J. IEEE Tans Microwave Theory Tech, 1999 ,47(4):433-442.

[5] Cameron R J. Advanced coupling matrix synthesis techniques for microwave filters, J. IEEE Tans Microwave Theory Tech, 2003,51:1-10. 